\title{
Some gregarious cycle decompositions of complete equipartite graphs
}

\author{
Benjamin R. Smith \\ Department of Mathematics \\ University of Queensland \\ Qld 4072, Australia \\ bsmith.maths@gmail.com
}

Submitted: Aug 17, 2009; Accepted: Nov 3, 2009; Published: Nov 13, 2009

Mathematics Subject Classification: 05C38, 05C51

\begin{abstract}
A $k$-cycle decomposition of a multipartite graph $G$ is said to be gregarious if each $k$-cycle in the decomposition intersects $k$ distinct partite sets of $G$. In this paper we prove necessary and sufficient conditions for the existence of such a decomposition in the case where $G$ is the complete equipartite graph, having $n$ parts of size $m$, and either $n \equiv 0,1(\bmod k)$, or $k$ is odd and $m \equiv 0(\bmod k)$. As a consequence, we prove necessary and sufficient conditions for decomposing complete equipartite graphs into gregarious cycles of prime length.
\end{abstract}

\section{Introduction and preliminaries}

We begin with some relevant definitions and terminology.

Let $K_{n}$ denote the complete graph on $n$ vertices and $\bar{K}_{n}$ denote the empty graph on $n$ vertices. For any graph $G$ and any positive integer $\lambda$, we denote the multigraph obtained from $G$ by replacing each of its edges with $\lambda$ parallel edges by $\lambda G$.

We denote the $k$-cycle containing edges $v_{1} v_{2}, v_{2} v_{3}, \ldots, v_{k-1} v_{k}$ and $v_{1} v_{k}$ by $\left(v_{1}, v_{2}, \ldots, v_{k}\right)$, or $\left(v_{k}, v_{k-1}, \ldots, v_{1}\right)$, or by any cyclic shift of these. We denote the $k$-path containing edges $v_{1} v_{2}, v_{2} v_{3}, \ldots, v_{k} v_{k+1}$ by $\left[v_{1}, v_{2}, \ldots, v_{k+1}\right]$ or $\left[v_{k+1}, v_{k}, \ldots, v_{1}\right]$ (hence, in our terminology, a $k$-path contains $k$ edges and $k+1$ vertices). Similarly, we denote the directed $k$-cycle containing directed edges $\left(v_{1}, v_{2}\right),\left(v_{2}, v_{3}\right), \ldots,\left(v_{k-1}, v_{k}\right)$ and $\left(v_{k}, v_{1}\right)$ by $\left(v_{1}, v_{2}, \ldots, v_{k}\right)_{D}$, or by any cyclic shift of this.

The lexicographic product $G * H$ of graphs $G$ and $H$ is the graph with vertex set $V(G) \times V(H)$, and with an edge joining $\left(g_{1}, h_{1}\right)$ to $\left(g_{2}, h_{2}\right)$ if and only if $g_{1} g_{2} \in E(G)$, or $g_{1}=g_{2}$ and $h_{1} h_{2} \in E(H)$. For our purposes we will primarily be concerned with 
lexicographic products of the form $G * \bar{K}_{m}$, for some $G$ and $m$. We note that for all graphs $G$ and positive integers $a$ and $\ell$,

$$
G * \bar{K}_{a \ell} \cong\left(G * \bar{K}_{a}\right) * \bar{K}_{\ell} \cong\left(G * \bar{K}_{\ell}\right) * \bar{K}_{a} .
$$

A subgraph of a multipartite graph $G$ is said to be gregarious in $G$ (or simply gregarious when $G$ is clear) if each of its vertices lies in a different partite set of $G$. In order to apply this definition, it must be clear what the partite sets of $G$ are. With this in mind we adopt the following convention.

Suppose $G$ is a graph on $n$ vertices, and $a$ and $\ell$ are positive integers. Throughout this paper, unless otherwise specified, we assume the graph $G * \bar{K}_{a \ell}$ has vertex set $\left\{v_{1}, v_{2}, \ldots, v_{a \ell} \mid v \in V(G)\right\}$, and an edge joining $u_{i}$ and $v_{j}$ in $G * \bar{K}_{a \ell}$ if and only if there is an edge joining $u$ and $v$ in $G$. The partite sets of $G * \bar{K}_{a \ell}$ are the $n$ sets $\left\{v_{1}, v_{2}, \ldots, v_{a \ell}\right\}$, $v \in V(G)$. However, we may occasionally choose to express the lexicographic product of $G$ and $\bar{K}_{a \ell}$ as $G * \bar{K}_{a(\ell)}$, or indeed $G * \bar{K}_{\ell(a)}$. In the first case, it is assumed that there is a further partitioning of each of the sets $\left\{v_{1}, v_{2}, \ldots, v_{a \ell}\right\}$ into $a$ subsets, each of size $\ell$. It is these $n a$ sets of size $\ell$ which are considered the partite sets of $G * \bar{K}_{a(\ell)}$. Similarly in the second case, it is assumed that there is a further partitioning of each of the sets $\left\{v_{1}, v_{2}, \ldots, v_{a \ell}\right\}$ into $\ell$ subsets, each of size $a$. It is these $n \ell$ sets of size $a$ which are considered the partite sets of $G * \bar{K}_{\ell(a)}$. Note that this means a given subgraph $H$ may be gregarious in $G * \bar{K}_{a(\ell)}$, but not gregarious in $G * \bar{K}_{a \ell}$.

A decomposition of a graph $G$ is a collection of subgraphs of $G$ whose edge-sets partition the edge-set of $G$. Let $\mathcal{H}=\left\{\mathcal{H}_{\infty}, \mathcal{H}_{\in}, \ldots, \mathcal{H}_{\sqcup}\right\}$ be a family of mutually nonisomorphic nontrivial graphs. An $\mathcal{H}$-decomposition of $G$ is a decomposition, $\mathcal{D}$ say, of $G$ such that

- for each $D \in \mathcal{D}$ there is some $H_{i} \in \mathcal{H}$ with $D \cong H_{i}$; and

- for each $H_{i} \in \mathcal{H}$ there is some $D \in \mathcal{D}$ with $H_{i} \cong D$.

If $\mathcal{H}=\{\mathcal{H}\}$ we often refer to such a decomposition as simply an $H$-decomposition. A decomposition of a multipartite graph $G$ is said to be gregarious if each of the subgraphs in the decomposition is gregarious in $G$.

In this paper we will be concerned with gregarious $k$-cycle decompositions of $K_{n} * \bar{K}_{m}$, the complete equipartite graph having $n$ parts of size $m$. Note that every vertex in $K_{n} * \bar{K}_{m}$ has degree $(n-1) m$ and the total number of edges in $K_{n} * \bar{K}_{m}$ is $n(n-1) m^{2} / 2$. Hence, obvious necessary conditions for the existence of such a decomposition are that

- $n \geqslant k \geqslant 3$;

- $(n-1) m$ is even; and

- $n(n-1) m^{2} \equiv 0(\bmod 2 k)$.

The study of gregarious cycle decompositions is relatively new and thus, compared to "nongregarious" cycle decompositions, few results are known. Of course 3-cycle decompositions of complete equipartite graphs are necessarily gregarious and so by Hanani [5], 
the above necessary conditions are sufficient when $k=3$. Sufficiency of these conditions has also been proved by Billington and Hoffman [2] when $k=4$, by Smith [9] when $k=5$ and by Billington, Hoffman and Smith [4] when $k \in\{6,8\}$. More generally, in [3] Billington, Hoffman and Rodger prove there exists a resolvable gregarious $n$-cycle decomposition of $K_{n} * \bar{K}_{m}$ if and only if $(n, m) \neq(3,2)$ or $(3,6)$; that is, the cycles in the decomposition can be partitioned into sets in such a way that the cycles in each set induce a 2-factor of $K_{n} * \bar{K}_{m}$. (Note that there exists nonresolvable gregarious 3-cycle decompositions of both $K_{3} * \bar{K}_{2}$ and $K_{3} * \bar{K}_{6}$.) In this paper, using the various new construction techniques presented in Section 2, we prove sufficiency of the above necessary conditions in cases where either $n \equiv 0,1(\bmod k)$, or $k$ is odd and $m \equiv 0(\bmod k)$. More formally, the main result (split into two parts) of this paper is the following.

Theorem 1.1 Suppose $n, m$ and $k$ are positive integers with $k \geqslant 3$. Necessary conditions for the existence of a gregarious $k$-cycle decomposition of $K_{n} * \bar{K}_{m}$ are that $n \geqslant k,(n-1) m$ is even and $n(n-1) m^{2} \equiv 0(\bmod 2 k)$.

(i) These conditions are sufficient whenever $n \equiv 0,1(\bmod k)$.

(ii) These conditions are sufficient whenever $k$ is odd and $m \equiv 0(\bmod k)$.

Part (i) of the above theorem is proved in Section 3, while part (ii) is proved in Section 4. Also, Theorem 1.1 has the following nice corollary.

Corollary 1.2 Suppose $n, m$ and $p$ are positive integers with $p$ an odd prime. Then there exists a gregarious p-cycle decomposition of $K_{n} * \bar{K}_{m}$ if and only if $n \geqslant p,(n-1) m$ is even and $n(n-1) m^{2} \equiv 0(\bmod 2 p)$.

Proof Since $n(n-1) m^{2} \equiv 0(\bmod 2 p)$ and $p$ is prime, we must have either $n \equiv 0,1$ $(\bmod p)$ or $m \equiv 0(\bmod p)$. The result then follows by Theorem 1.1.

Hence, for arbitrary $n$ and $m$, Corollary 1.2 gives the first known infinite family of values of $k$ for which the obvious necessary conditions for the existence of a gregarious $k$-cycle decomposition of $K_{n} * \bar{K}_{m}$ are also sufficient.

\section{Some new decomposition techniques}

In this section we introduce some new techniques for obtaining gregarious cycle decompositions of complete equipartite graphs from cycle decompositions of related multigraphs. The techniques used are similar to those introduced in [11].

We begin with the following definition.

Definition 2.1 Suppose $\mathcal{D}=\left\{H_{1}, H_{2}, \ldots, H_{t}\right\}$ is a decomposition of $\lambda G$. A $\lambda$-weight function on $\mathcal{D}$ is any function $\omega$ which assigns an integer label, from the set $\{0,1, \ldots, \lambda-1\}$, to each edge of the graphs $H_{1}, H_{2}, \ldots, H_{t}$, in such a way that distinct copies of the same edge receive distinct labels. (Hence for each $\ell \in\{0,1, \ldots, \lambda-1\}$, the edges labelled $\ell$ induce a copy of the graph $G$.) 
Note that the above definition is a specialisation of a more general type of "weight function" first described in [11].

The next lemma shows how a cycle decomposition of the graph $2 G$, together with a 2-weight function, can be used to generate a gregarious cycle decomposition of the graph $G * \bar{K}_{2}$ when certain extra conditions on the original decomposition are satisfied.

Lemma 2.2 Suppose there exists a k-cycle decomposition of $2 G$ which can be partitioned into pairs of cycles in such a way that the cycles in each pair share two adjacent edges. Then there exists a gregarious $k$-cycle decomposition of $G * \bar{K}_{2}$.

Proof Let $\mathcal{D}$ be a $k$-cycle decomposition of $2 G$ which satisfies the conditions of the lemma and let $\omega$ be any 2-weight function on $\mathcal{D}$. Recall that $G * \bar{K}_{2}$ is the graph obtained from $G$ by replacing each vertex $v$ in $G$ with the set of vertices $\left\{v_{1}, v_{2}\right\}$, and each edge $u v$ in $G$ with the edges $u_{1} v_{1}, u_{1} v_{2}, u_{2} v_{1}$ and $u_{2} v_{2}$.

For each $k$-cycle $C \in \mathcal{D}$ we generate a subgraph, denoted by $\hat{C}$, of $G * \bar{K}_{2}$ by associating each vertex $v$ in $C$ with the set of vertices $\left\{v_{1}, v_{2}\right\}$, and each edge $u v$ in $C$ having label $\ell$ (under the function $\omega$ ) with the pair of edges $u_{1} v_{1+\ell}$ and $u_{2} v_{2+\ell}$ (subscripts calculated mod 2). Note that $\hat{C}$ is either a single $2 k$-cycle (if $C$ contains an odd number of edges labelled 1), or two vertex disjoint $k$-cycles which are gregarious in $G * \bar{K}_{2}$ (if $C$ contains an even number of edges labelled 1). Since $\omega$ is a 2-weight function, the graphs $\hat{C}$ together decompose $G * \bar{K}_{2}$. Hence we need only show that, if $C$ and $C^{\prime}$ are any "pair" of cycles from the partition of $\mathcal{D}$, then the graph $\hat{C} \cup \hat{C}^{\prime}$ admits a decomposition into $k$-cycles which are gregarious in $G * \bar{K}_{2}$.

Let $u v$ and $v w$ be the two adjacent edges shared by $C$ and $C^{\prime}$. Then $\hat{C} \cup \hat{C}^{\prime}$ contains the edges $u_{i} v_{j}$ and $v_{i} w_{j}$ for each $i, j \in\{1,2\}$. Let $H$ be the subgraph of $\hat{C} \cup \hat{C}^{\prime}$ spanned by these edges, and let $H^{\prime}$ be the complement of $H$ in $\hat{C} \cup \hat{C}^{\prime}$. (Hence $\left\{H, H^{\prime}\right\}$ is a decomposition of $\hat{C} \cup \hat{C}^{\prime}$.) Then for some $a, b \in\{1,2\}$ the graph $H^{\prime}$ decomposes into four gregarious $(k-2)$-paths:

$$
L_{1}=\left[w_{a}, \ldots, u_{1}\right] ; \quad L_{2}=\left[w_{a+1}, \ldots, u_{2}\right] ; \quad L_{3}=\left[w_{b}, \ldots, u_{1}\right] ; \quad L_{4}=\left[w_{b+1}, \ldots, u_{2}\right] .
$$

Now $H$ decomposes into the four gregarious 2-paths:

$$
P_{1}=\left[u_{1}, v_{1}, w_{a}\right] ; \quad P_{2}=\left[u_{2}, v_{1}, w_{a+1}\right] ; \quad P_{3}=\left[u_{1}, v_{2}, w_{b}\right] ; \quad P_{4}=\left[u_{2}, v_{2}, w_{b+1}\right] .
$$

The result then follows by adjoining the $(k-2)$-path $L_{i}$ to the 2-path $P_{i}$, for each $i \in$ $\{1,2,3,4\}$.

Note that the "pairing" condition in Lemma 2.2 means there must be an even number of cycles in the decomposition of $2 G$. In fact, it is easy to see that we can relax this condition slightly and obtain the following generalisation.

Lemma 2.3 Suppose there exists a $k$-cycle decomposition of $2 G$ which can be partitioned into two parts, say $\mathcal{D}_{1}$ and $\mathcal{D}_{2}$, so that every cycle in $\mathcal{D}_{1}$ shares an edge with some cycle in $\mathcal{D}_{2}$, and $\mathcal{D}_{2}$ can be partitioned into pairs of cycles in such a way that the cycles in each pair share two adjacent edges. Then there exists a gregarious $k$-cycle decomposition of $G * \bar{K}_{2}$. 
Proof Let $\mathcal{D}=\mathcal{D}_{1} \cup \mathcal{D}_{2}$. We show there exists a 2-weight function $\omega$ on $\mathcal{D}$ with the property that each cycle in $\mathcal{D}_{1}$ contains an even number of edges labelled 1 (under $\omega$ ). Using the notation defined in the proof of Lemma 2.2, the result then follows since for each $C \in \mathcal{D}_{1}$, the graph $\hat{C}$ consists of two $k$-cycles which are gregarious in $G * \bar{K}_{2}$, and for each "pair" of cycles $C$ and $C^{\prime}$ in the partition of $\mathcal{D}_{2}$ the graph $\hat{C} \cup \hat{C}^{\prime}$ admits a decomposition into $k$-cycles which are gregarious in $G * \bar{K}_{2}$ (using the method described in the proof of Lemma 2.2).

Let $\omega$ be any 2 -weight function on $\mathcal{D}$. If each cycle in $\mathcal{D}_{1}$ contains an even number of edges labelled 1 we are done. If not, we modify $\omega$ as follows. Suppose $C \in \mathcal{D}_{1}$ and $C$ contains an odd number of edges labelled 1 . Now $C$ contains an edge, $e$ say, which is also contained in some cycle, $C^{\prime}$ say, in $\mathcal{D}_{2}$. We switch the label on the edge $e$ in $C$ from either 0 to 1 , or vice-versa. We then do the same for the copy of the edge $e$ in $C^{\prime}$. Hence the resulting edge labelling still induces a 2 -weight function on $\mathcal{D}$. Moreover, the cycle $C$ now contains an even number of edges labelled 1 and we have not affected the edge labellings of any other cycles in $\mathcal{D}_{1}$. We repeat this process for each cycle in $\mathcal{D}_{1}$ having an odd number of edges labelled 1 . The resulting edge labelling then induces a 2 -weight function on $\mathcal{D}$ with the required property.

In order to more fully exploit these newly defined $\lambda$-weight functions we also introduce the idea of the "sum-weight" of a cycle. (Again, this concept was originally defined in [11].)

Definition 2.4 Suppose $C$ is a cycle in the graph $G$. Suppose furthermore that $\vec{G}$ is the graph obtained by orienting the edges of $G$ in some way, and that $\omega$ is a function which assigns an integer label $\omega(e)$ to each edge $e$ in the cycle $C$. We let $\vec{C}$ be a directed cycle formed by orienting the edges of $C$, and for each edge $e$ in $C$ we denote the corresponding directed edge in $\vec{C}$ by $e^{\prime}$. (Note that there are two possible choices for $\vec{C}$, and that $\vec{C}$ need not be a subgraph of $\vec{G}$.) The sum-weight with respect to $\vec{G}$ under the function $\omega$ of the cycle $C$, is then defined to be the absolute value of the sum, over all edges $e$ in $C$, of $f\left(e^{\prime}\right) \omega(e)$, where

$$
f\left(e^{\prime}\right)= \begin{cases}1, & \text { if } e^{\prime} \text { is an edge in } \vec{G} \\ -1, & \text { otherwise }\end{cases}
$$

Note that taking the "absolute value" ensures that the sum-weight is independent of the choice of $\vec{C}$. Furthermore, when both $\vec{G}$ and $\omega$ are clear, we will often refer simply to the sum-weight of the cycle $C$, rather than the sum-weight with respect to $\vec{G}$ under the function $\omega$.

In [11] we defined the notion of an unbalanced $\lambda$-weight function on a cycle decomposition of a graph $\lambda G$. These functions have the property that under some particular orientation of the edges of $G$, say $\vec{G}$, each cycle in the decomposition of $\lambda G$ has sum-weight coprime to $\lambda$. Hence each $k$-cycle $C$ in the decomposition of $\lambda G$ can be used to generate a $\lambda k$-cycle in the graph $G * \bar{K}_{\lambda}$ by associating each vertex $v$ in $C$ with the partite set $A_{v}=\left\{v_{1}, v_{2}, \ldots, v_{\lambda}\right\}$, and each edge $u v$ labelled $\ell$ in $C$ with the matching between $A_{u}$ 
and $A_{v}$ consisting of the edges $u_{1} v_{1+\ell}, u_{2} v_{2+\ell}, \ldots, u_{\lambda} v_{\lambda+\ell}$ if $(u, v)$ is an edge in $\vec{G}$, or the edges $v_{1} u_{1+\ell}, v_{2} u_{2+\ell}, \ldots, v_{\lambda} u_{\lambda+\ell}$ if $(v, u)$ is an edge in $\vec{G}$. The fact that the sum-weight of $C$ is coprime to $\lambda$ ensures that the $k$ matchings form a single $\lambda k$-cycle in $G * \bar{K}_{\lambda}$, rather than a collection of disjoint cycles whose lengths sum to $\lambda k$. Since here we are interested in gregarious cycle decompositions, we will instead be concerned with $\lambda$-weight functions under which each cycle in a decomposition of $\lambda G$ has sum-weight a multiple of $\lambda$. In this case, a $k$-cycle in the decomposition of $\lambda G$ will generate, using the same method as described above, a subgraph in $G * \bar{K}_{\lambda}$ consisting of $\lambda$ pairwise vertex-disjoint gregarious $k$-cycles. We call such a function a balanced $\lambda$-weight function. More formally we define this as follows.

Definition 2.5 Suppose $\mathcal{D}$ is a cycle decomposition of $\lambda G$ and $\omega$ is a $\lambda$-weight function on $\mathcal{D}$. Then $\omega$ is said to be balanced if, under some orientation of the edges of $G$, each cycle in $\mathcal{D}$ has sum-weight a multiple of $\lambda$.

The following lemma proves that such functions do indeed generate gregarious cycle decompositions.

Lemma 2.6 Suppose $\mathcal{D}$ is a $k$-cycle decomposition of $\lambda G$ and $\omega$ is a balanced $\lambda$-weight function on $\mathcal{D}$. Then there exists a gregarious $k$-cycle decomposition of $G * \bar{K}_{\lambda}$.

Proof Let $\vec{G}$ be the particular orientation of the edges of $G$ under which $\omega$ is balanced. For each $k$-cycle $C \in \mathcal{D}$ we generate a subgraph in $G * \bar{K}_{\lambda}$ by associating each vertex $v$ in $C$ with the partite set $A_{v}=\left\{v_{1}, v_{2}, \ldots, v_{\lambda}\right\}$, and each edge $u v$ in $C$ having label $\ell$ (under the function $\omega$ ) with the matching between partite sets $A_{u}$ and $A_{v}$ consisting of the edges $u_{1} v_{1+\ell}, u_{2} v_{2+\ell}, \ldots, u_{\lambda} v_{\lambda+\ell}$ if $(u, v)$ is an edge in $\vec{G}$, or the edges $v_{1} u_{1+\ell}, v_{2} u_{2+\ell}, \ldots, v_{\lambda} u_{\lambda+\ell}$ if $(v, u)$ is an edge in $\vec{G}$. Hence each of these subgraphs is a 2-regular graph on $\lambda k$ vertices. In fact, since the sum-weight of each cycle in $\mathcal{D}$ is a multiple of $\lambda$, each of these subgraphs necessarily consists of $\lambda$ pairwise vertex-disjoint $k$-cycles, each of which is gregarious in $G * \bar{K}_{\lambda}$. Furthermore, since $\omega$ is a $\lambda$-weight function, the collection of all such $k$-cycles forms a decomposition of $G * \bar{K}_{\lambda}$ as required.

\section{The case $n \equiv 0,1(\bmod k)$}

The aim of this section is to prove Theorem 1.1 (i). We make extensive use of the following three obvious, but surprisingly useful, results.

Lemma 3.1 Suppose there exists a gregarious $\left\{H_{1}, H_{2}, \ldots, H_{t}\right\}$-decomposition of $G * \bar{K}_{a}$ and, for each $i \in\{1,2, \ldots, t\}$, there exists a gregarious $H$-decomposition of $H_{i} * \bar{K}_{\ell}$. Then there exists a gregarious $H$-decomposition of $G * \bar{K}_{a \ell}$.

Lemma 3.2 Suppose there exists a $\left\{H_{1}, H_{2}, \ldots, H_{t}\right\}$-decomposition of $G * \bar{K}_{a}$ and, for each $i \in\{1,2, \ldots, t\}$, there exists a gregarious $H$-decomposition of $H_{i} * \bar{K}_{\ell}$. Then there exists a gregarious $H$-decomposition of $G * \bar{K}_{a(\ell)}$. 
Lemma 3.3 Suppose there exists a gregarious $\left\{H_{1}, H_{2}, \ldots, H_{t}\right\}$-decomposition of $G * \bar{K}_{a}$ and, for each $i \in\{1,2, \ldots, t\}$, there exists an $H$-decomposition of $H_{i} * \bar{K}_{\ell}$. Then there exists a gregarious $H$-decomposition of $G * \bar{K}_{\ell(a)}$.

The following is a direct application of Lemma 3.2.

Lemma 3.4 Suppose there exists a k-cycle decomposition of $G * \bar{K}_{a}$. Then, for each positive integer $\ell$, there exists a gregarious $k$-cycle decomposition of $G * \bar{K}_{a(\ell)}$.

Proof By Lemma 3.2 we need only prove there exists a gregarious $k$-cycle decomposition of $C * \bar{K}_{\ell}$, where $C$ is a (generic) $k$-cycle. Suppose $C=(1,2, \ldots, k)$. If $\ell$ is even we take the $\ell^{2}$ cycles $\left(1_{i}, 2_{j}, \ldots,(k-1)_{i}, k_{j}\right)$, where $i, j \in\{1,2, \ldots, \ell\}$. If $\ell$ is odd we take the $\ell^{2}$ cycles $\left(1_{i}, 2_{j}, \ldots,(k-1)_{j}, k_{i \circ j}\right)$, where $i, j \in\{1,2, \ldots, \ell\}$ and $i \circ j$ is the entry in row $i$ and column $j$ of any (fixed) latin square of order $\ell$ on the set $\{1,2, \ldots, \ell\}$. It is an easy exercise to check that these cycles decompose $C * \bar{K}_{\ell}$ as required.

We can apply Lemma 3.1 in a similar way and obtain the following easy result.

Lemma 3.5 Suppose there exists a gregarious $k$-cycle decomposition of $G * \bar{K}_{a}$. Then, for each positive integer $\ell$, there exists a gregarious $k$-cycle decomposition of $G * \bar{K}_{a \ell}$.

We will also make use of some well-known results involving cycle decompositions of complete graphs and complete equipartite graphs. The first such result, due to Alspach, Gavlas [1] and Săjna [8], gives necessary and sufficient conditions for the existence of a $k$-cycle decomposition of the complete graph $K_{n}$.

Theorem 3.6 ([1],[8]) Suppose $n$ and $k$ are positive integers with $n \geqslant 3$ and $k \geqslant 3$. Then there exists a $k$-cycle decomposition of $K_{n}$ if and only if $n \geqslant k, n$ is odd and $n(n-1) \equiv 0$ $(\bmod 2 k)$.

We note that if there exists a $k$-cycle decomposition of $K_{n}$ then, by Lemma 3.4, for each positive integer $m$ there exists a gregarious $k$-cycle decomposition of $K_{n} * \bar{K}_{m}$. Hence we have the following obvious corollary to Theorem 3.6.

Corollary 3.7 Suppose $n, m$ and $k$ are positive integers with $n \geqslant 3$ and $k \geqslant 3$. Then there exists a gregarious $k$-cycle decomposition of $K_{n} * \bar{K}_{m}$ whenever $n \geqslant k, n$ is odd and $n(n-1) \equiv 0(\bmod 2 k)$.

The next theorem follows from a stronger result of Liu [6],[7] involving resolvable cycle decompositions of complete equipartite graphs. Note that we have removed the "resolvability" condition from Liu's original result since we will not be concerned with that property here (this also allows us to easily remove the "exceptions" from Liu's original result).

Theorem 3.8 ([6],[7]) Suppose $n, m$ and $k$ are positive integers with $n \geqslant 3$ and $k \geqslant 3$. Then there exists a $k$-cycle decomposition of $K_{n} * \bar{K}_{m}$ whenever $(n-1) m$ is even and $n m \equiv 0(\bmod k)$. 
Combining this result with Lemma 3.4 we have the following easy corollary.

Corollary 3.9 Suppose $n, \ell, a$ and $k$ are positive integers with $n \geqslant 3$ and $k \geqslant 3$. Then there exists a gregarious $k$-cycle decomposition of $K_{n} * \bar{K}_{a k(\ell)}$ whenever $(n-1)$ ak is even.

We now state a result of Billington et al. [3] involving gregarious $k$-cycle decompositions of $K_{k} * \bar{K}_{m}$. We note, as mentioned in the introduction, that they actually proved necessary and sufficient conditions for the existence of a resolvable gregarious $k$-cycle decomposition of $K_{k} * \bar{K}_{m}$ however, as already noted, we will not be concerned with this additional property here.

Theorem 3.10 ([3]) Suppose $k$ and $m$ are positive integers with $k \geqslant 3$. Then there exists a gregarious $k$-cycle decomposition of $K_{k} * \bar{K}_{m}$ if and only if either $k$ is odd or $m$ is even.

This result has the following simple corollary in the case that $k$ is even.

Corollary 3.11 Suppose $n, m$ and $k$ are positive integers with $m$ and $k$ even, and $k \geqslant 4$. Then there exists a gregarious $k$-cycle decomposition of $K_{n} * \bar{K}_{m}$ whenever $n \equiv 0(\bmod k)$.

Proof If $n=k$ the result follows immediately by Theorem 3.10. Suppose then that $n=q k$, with $q \geqslant 2$. It is easy to see that there exists a $\left\{K_{k}, K_{q} * \bar{K}_{k}\right\}$-decomposition of $K_{n} \cong K_{q k} * \bar{K}_{1}$. Moreover, there exist gregarious $k$-cycle decompositions of $K_{k} * \bar{K}_{m}$, by Theorem 3.10, and of $K_{q} * \bar{K}_{k(m)}$, by Corollary 3.9. Hence the result follows by Lemma 3.2 .

Using the techniques developed in Section 1 (in particular Lemma 2.3), we now present a series of three lemmas (each followed immediately by a corollary) in which we obtain some useful gregarious cycle decompositions of complete equipartite graphs having parts of even size. The first of these decompositions deals with cases in which the cycle length is also even.

Lemma 3.12 Suppose $k$ and $m$ are positive integers with $k \geqslant 4$. Then there exists a gregarious $k$-cycle decomposition of $K_{k+1} * \bar{K}_{m}$ whenever $k$ and $m$ are both even.

Proof If $k=4$ the result follows from [2]. Suppose then $k \geqslant 6$. Note that we need only consider the case $m=2$ and the result then follows by Lemma 3.5. Furthermore, in the case $m=2$ we need only give a $k$-cycle decomposition of $2 K_{k+1}$ which satisfies the conditions of Lemma 2.3. We do this as follows.

Let the vertex set of $2 K_{k+1}$ be $\mathbb{Z}_{k} \cup\{\infty\}$, and let $\rho=(012 \cdots(k-1))(\infty)$ be a permutation of order $k$ on $V\left(2 K_{k+1}\right)$. We define the $k$-cycles $C$ and $D$ on $2 K_{k+1}$ by

$$
\begin{aligned}
& C=(0,1, k-1,2, k-2, \ldots, k / 2-1, k / 2+1, \infty) ; \text { and } \\
& D=(0,1,2, \ldots, k-1) .
\end{aligned}
$$

(See for example Figure 1, which shows the cycles $C$ and $D$ in the case $k=16$.) 

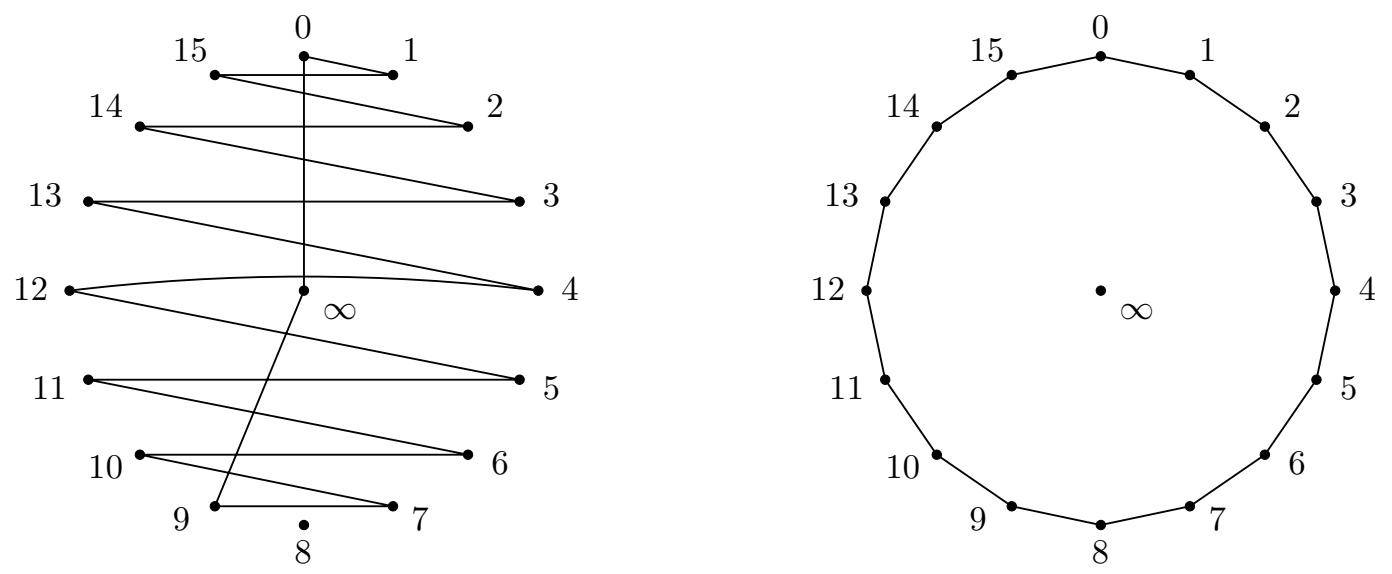

Figure 1: The cycles $C$ and $D$ when $k=16$

It is a simple exercise to check that $\mathcal{D}=\left\{D, \rho^{0}(C), \rho^{1}(C), \ldots, \rho^{k-1}(C)\right\}$ is a $k$-cycle decomposition of $2 K_{k+1}$. We note that, for each $i \in\{0,1, \ldots, k / 2-1\}$, the cycles $\rho^{i}(C)$ and $\rho^{i+k / 2}(C)$ both contain the 2-path $[i+1, i-1, i+2]$. Hence, setting $\mathcal{D}_{1}=\{D\}$ and $\mathcal{D}_{2}=\left\{\rho^{0}(C), \rho^{1}(C), \ldots, \rho^{k-1}(C)\right\}$, it is easy to see that $\mathcal{D}$ satisfies the conditions of Lemma 2.3 and the result follows.

Corollary 3.13 Suppose $n, m$ and $k$ are positive integers with $m$ and $k$ even, and $k \geqslant 4$. Then there exists a gregarious $k$-cycle decomposition of $K_{n} * \bar{K}_{m}$ whenever $n \geqslant k$ and $n \equiv 1(\bmod k)$.

Proof If $n=k+1$ the result follows immediately by Lemma 3.12. Suppose then that $n=q k+1$, with $q \geqslant 2$. It is easy to see that there exists a $\left\{K_{k+1}, K_{2} * \bar{K}_{k}\right\}$-decomposition of $K_{n} \cong K_{q k+1} * \bar{K}_{1}$. Moreover, there exist gregarious $k$-cycle decompositions of $K_{k+1} * \bar{K}_{m}$, by Lemma 3.12, and of $K_{2} * \bar{K}_{k(m)}$, by Corollary 3.9. Hence the result follows by Lemma 3.2 .

The next result is analogous to that of Lemma 3.12 in the case that $k$ is odd.

Lemma 3.14 Suppose $k$ and $m$ are positive integers with $k \geqslant 3$. Then there exists a gregarious $k$-cycle decomposition of $K_{k+1} * \bar{K}_{m}$ whenever $k$ is odd and $m$ is even.

Proof As in the proof of Lemma 3.12, we need only give a $k$-cycle decomposition of $2 K_{k+1}$ which satisfies the conditions of Corollary 2.3. The result then follows for $m=2$ by Lemma 2.3, and subsequently for all even $m$ by Lemma 3.5.

Let the vertex set of $2 K_{k+1}$ be $\mathbb{Z}_{k} \cup\{\infty\}$, and let $\rho=(012 \cdots(k-1))(\infty)$ be a permutation of order $k$ on $V\left(2 K_{k+1}\right)$. We then split the problem according to the congruence of $k$ modulo 4 . 
Case I. Suppose $k \equiv 1(\bmod 4)$. Let $k=4 \ell+1$ and define the $(4 \ell+1)$-cycles $C$ and $D$ on $2 K_{4 \ell+2}$ by

$$
\begin{aligned}
& C=(0,1,4 \ell, 2,4 \ell-1, \ldots, \ell, 3 \ell+1, \ell+2,3 \ell, \ell+3, \ldots, 2 \ell, 2 \ell+2,2 \ell+1, \infty) ; \text { and } \\
& D=(0,2 \ell, 4 \ell, 2 \ell-1,4 \ell-1, \ldots, 1,2 \ell+1) .
\end{aligned}
$$

(See for example Figure 2, which shows the cycles $C$ and $D$ in the case $k=17$.)
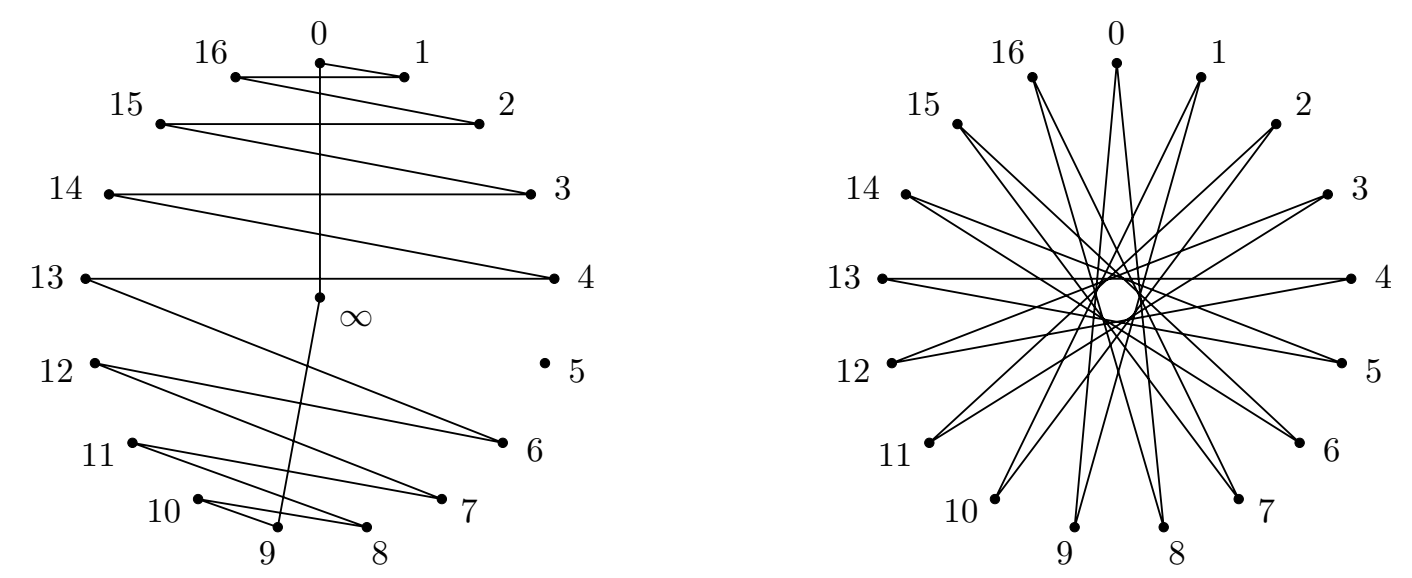

Figure 2: The cycles $C$ and $D$ when $k=17$

It is a simple exercise to check that $\mathcal{D}=\left\{D, \rho^{0}(C), \rho^{1}(C), \ldots, \rho^{4 \ell}(C)\right\}$ is a $k$-cycle decomposition of $2 K_{4 \ell+2}$. We note that, for each $i \in\{0,1, \ldots, 2 \ell-1\}$, the cycles $\rho^{i}(C)$ and $\rho^{i+2 \ell}(C)$ both contain the 2-path $[\infty, i, i+1]$. Hence, setting $\mathcal{D}_{1}=\left\{D, \rho^{4 \ell}(C)\right\}$ and $\mathcal{D}_{2}=\left\{\rho^{0}(C), \rho^{1}(C), \ldots, \rho^{4 \ell-1}(C)\right\}$, it is easy to see that $\mathcal{D}$ satisfies the conditions of Lemma 2.3 and the result follows.

Case II. Suppose $k \equiv 3(\bmod 4)$. Let $k=4 \ell+3$ and define the $(4 \ell+3)$-cycles $C$ and $D$ on $2 K_{4 \ell+4}$ by

$C=(0,1,4 \ell+2,2,4 \ell+1, \ldots, 3 \ell+3, \ell+1,3 \ell+1, \ell+2,3 \ell, \ldots, 2 \ell, 2 \ell+2,2 \ell+1, \infty) ;$ and $D=(0,2 \ell+1,4 \ell+2,2 \ell, 4 \ell+1, \ldots, 1,2 \ell+2)$.

It is a simple exercise to check that $\mathcal{D}=\left\{D, \rho^{0}(C), \rho^{1}(C), \ldots, \rho^{4 \ell+2}(C)\right\}$ is a $k$-cycle decomposition of $2 K_{4 \ell+4}$. We note that, for each $i \in\{0,1,2, \ldots, 2 \ell-1\}$, the cycles $\rho^{i}(C)$ and $\rho^{i+2 \ell+2}(C)$ both contain the 2-path $[\infty, i, i+1]$. Hence, setting $\mathcal{D}_{1}=\left\{D, \rho^{2 \ell+1}(C)\right\}$ and $\mathcal{D}_{2}=\left\{\rho^{0}(C), \rho^{1}(C), \ldots, \rho^{2 \ell}(C), \rho^{2 \ell+2}(C), \rho^{2 \ell+3}(C), \ldots, \rho^{4 \ell+2}(C)\right\}$, it is easy to see that $\mathcal{D}$ satisfies the conditions of Lemma 2.3 and the result follows.

Corollary 3.15 Suppose $n, m$ and $k$ are positive integers with $n$ and $m$ even, $k$ odd and $k \geqslant 3$. Then there exists a gregarious $k$-cycle decomposition of $K_{n} * \bar{K}_{m}$ whenever $n \geqslant k$ and $n \equiv 1(\bmod k)$. 
Proof If $k=n-1$, the result follows immediately from Lemma 3.14. Suppose then $n=q k+1$, with $q \geqslant 3$ and $q$ odd (since $n$ is even). It is easy to see that there exists a $\left\{K_{k+1}, K_{q} * \bar{K}_{k}\right\}$-decomposition of $K_{n} \cong K_{q k+1} * \bar{K}_{1}$. Moreover, there exist gregarious $k$-cycle decompositions of $K_{k+1} * \bar{K}_{m}$, by Lemma 3.14 , and of $K_{q} * \bar{K}_{k(m)}$, by Corollary 3.9. Hence the result follows by Lemma 3.2.

The third and final lemma will be used to give the analogous result to Corollary 3.11 in the case that $k$ is odd.

Lemma 3.16 Suppose $k$ and $m$ are positive integers with $k \geqslant 3$. Then there exists a gregarious $k$-cycle decomposition of $K_{2 k} * \bar{K}_{m}$ whenever $k$ is odd and $m$ is even.

Proof The case $k=3$ (in which all cycles are necessarily gregarious) was settled by Hanani [5]. Assume then $k \geqslant 5$.

Similar to the proof of Lemma 3.14, we need only give a decomposition of $2 K_{2 k}$ into $k$-cycles which satisfies the conditions of Lemma 2.2. The result then follows for $m=2$ by Lemma 2.2, and subsequently for all even $m$ by Lemma 3.5.

Let the vertex set of $2 K_{2 k}$ be $\mathbb{Z}_{2 k-1} \cup\{\infty\}$, and let $\rho=(012 \cdots(2 k-2))(\infty)$ be a permutation of order $2 k-1$ on $V\left(2 K_{2 k}\right)$. We then split the problem according to the congruence of $k$ modulo 4 .

Case I. Suppose $k \equiv 1(\bmod 4)$. Let $k=4 \ell+1$, then $\ell \geqslant 1$ and $2 k-1=8 \ell+1$. Define the $(4 \ell+1)$-cycles $C$ and $D$ on $2 K_{8 \ell+2}$ by

$$
\begin{aligned}
& C=(0,1,8 \ell, 2,8 \ell-1, \ldots, 6 \ell+2,2 \ell, 6 \ell) ; \text { and } \\
& D=(0,1,8 \ell, 2,8 \ell-1, \ldots, 7 \ell+1, \ell+2,7 \ell, \ell+3,7 \ell-1, \ldots, 6 \ell+2,2 \ell+1, \infty) .
\end{aligned}
$$

(See for example Figure 3, which shows the cycles $C$ and $D$ in the case $k=9$.)
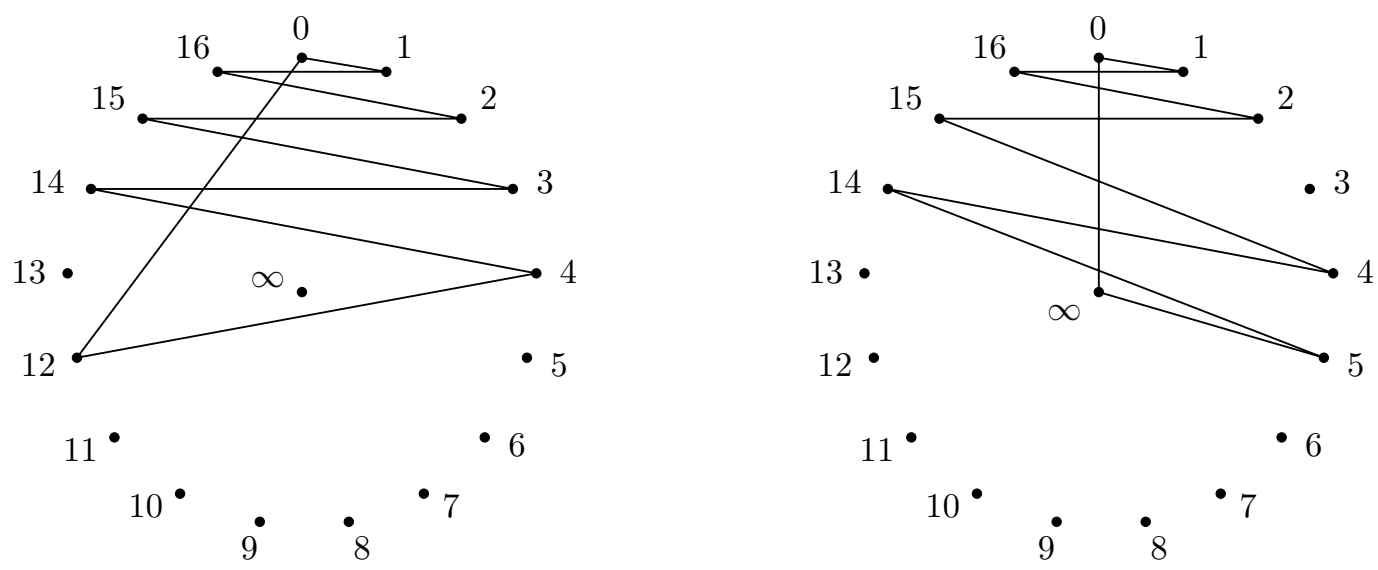

Figure 3: The cycles $C$ and $D$ when $k=9$

It is a simple exercise to check that $\mathcal{D}=\left\{\rho^{i}(C), \rho^{i}(D) \mid 0 \leqslant i \leqslant 2 k-2\right\}$ is a $k$-cycle decomposition of $2 K_{8 \ell+2}$. We note that the cycles $C$ and $D$ both contain the 2-path 
$[0,1,8 \ell]$. Hence it is easy to see that $\mathcal{D}$ satisfies the conditions of Lemma 2.2 and the result follows.

Case II. Suppose $k \equiv 3(\bmod 4)$. Let $k=4 \ell+3$, then $\ell>1$ and $2 k-1=8 \ell+5$. Define the $(4 \ell+3)$-cycles $C$ and $D$ on $2 K_{8 \ell+6}$ by

$$
\begin{aligned}
& C=(0,1,8 \ell+4,2,8 \ell+3, \ldots, 6 \ell+4,2 \ell+1,6 \ell+3) ; \text { and } \\
& D=(0,1,8 \ell+4,2,8 \ell+3, \ldots, \ell, 7 \ell+5, \ell+2,7 \ell+4, \ell+3, \ldots, 6 \ell+4,2 \ell+2, \infty) .
\end{aligned}
$$

It is a simple exercise to check that $\mathcal{D}=\left\{\rho^{i}(C), \rho^{i}(D) \mid 0 \leqslant i \leqslant 2 k-2\right\}$ is a $k$-cycle decomposition of $2 K_{8 \ell+2}$. We note that the cycles $C$ and $D$ both contain the 2-path $[0,1,8 \ell+4]$. Hence it is easy to see that $\mathcal{D}$ satisfies the conditions of Lemma 2.2 and the result follows.

Corollary 3.17 Suppose $n, m$ and $k$ are positive integers with $n$ and $m$ even, $k$ odd and $k \geqslant 3$. Then there exists a gregarious $k$-cycle decomposition of $K_{n} * \bar{K}_{m}$ whenever $n \geqslant k$ and $n \equiv 0(\bmod k)$.

Proof If $n=2 k$ the result follows immediately from Lemma 3.16. If $n=4 k$ it is easy to see that there exists a $\left\{K_{k}, K_{4} * \bar{K}_{k}\right\}$-decomposition of $K_{n} \cong K_{4 k} * \bar{K}_{1}$. Moreover, there exists a gregarious $k$-cycle decomposition of $K_{k} * \bar{K}_{m}$, by Theorem 3.10, and there exists a gregarious $k$-cycle decomposition of $K_{4} * \bar{K}_{k(m)}$ as follows. Since $m$ is even, there exists a gregarious $K_{3}$-decomposition of $K_{4} * \bar{K}_{m}$ by Hanani [5]. Furthermore, there exists a $k$-cycle decomposition of $K_{3} * \bar{K}_{k}$ by Theorem 3.8. Hence there exists a gregarious $k$-cycle decomposition of $K_{4} * \bar{K}_{k(m)}$, by Lemma 3.3. The result then follows by Lemma 3.2. Assume then $n=2 q k$ for some $q \geqslant 3$.

It is easy to see that there exists a $\left\{K_{2 k}, K_{q} * \bar{K}_{2 k}\right\}$-decomposition of $K_{n} \cong K_{2 q k} * \bar{K}_{1}$. Moreover, there exist gregarious $k$-cycle decompositions of $K_{2 k} * \bar{K}_{m}$, by Theorem 3.16, and of $K_{q} * \bar{K}_{2 k(m)}$, by Corollary 3.9. Hence the result follows by Lemma 3.2.

We are now ready to present the proof of Theorem 1.1 (i).

Proof of Theorem 1.1 (i) Let $n, m$ and $k$ satisfy the conditions of the theorem. We note first that if $n$ and $m$ are both odd and $n \equiv 1(\bmod k)$, then $n \equiv 1(\bmod 2 k)$ since $n(n-1) m^{2} \equiv 0(\bmod 2 k)$. We then split the problem according to the parity of $k$ and whether $n \equiv 0$ or $1(\bmod k)$.

Case I. Suppose $k$ is even and $n \equiv 0(\bmod k)$. We note that $n$ is even and hence $m$ is also even. The result then follows by Corollary 3.11.

Case II. Suppose $k$ is even and $n \equiv 1(\bmod k)$. If $m$ is even the result follows by Corollary 3.13. If $m$ is odd then $n$ is odd and, as noted above, $n \equiv 1(\bmod 2 k)$. The result then follows by Corollary 3.7 .

Case III. Suppose $k$ is odd and $n \equiv 0(\bmod k)$. If $n$ is odd the result follows by Corollary 3.7. Otherwise, both $n$ and $m$ are even and the result follows by Corollary 3.17. 
Case IV. Suppose $k$ is odd and $n \equiv 1(\bmod k)$. If $n$ is odd the result follows by Corollary 3.7. Otherwise, both $n$ and $m$ are even and the result follows by Corollary 3.15.

This completes the proof.

\section{The case $m \equiv 0(\bmod k)$}

The aim of this section is to prove Theorem 1.1 (ii). The critical ingredient in our proof is the following lemma.

Lemma 4.1 Suppose $n$ and $\lambda$ are odd with $n \geqslant \lambda \geqslant 3$. Then:

(i) there exists a $\lambda$-cycle decomposition of $\lambda K_{n}$ with balanced $\lambda$-weight function $\omega$; and

(ii) if $\lambda \geqslant 5$, there exists a gregarious $\lambda$-cycle decomposition of $2\left(K_{n+1} * \bar{K}_{\lambda}\right)$ which can be partitioned into pairs in such a way that the cycles in each pair share two adjacent edges.

Proof Note that the following $\lambda$-cycle decomposition of $\lambda K_{n}$ was first given by the author in [10].

Let $n=2 t+1, \lambda=2 \mu+1$, the vertex set of $\lambda K_{n}$ be $\mathbb{Z}_{2 t+1}$ and $\rho=\left(\begin{array}{ll}0 & 1 \cdots 2 t) \text { be }\end{array}\right.$ a permutation of order $2 t+1$ on $V\left(\lambda K_{2 t+1}\right)$. We define the difference of an edge $u v$ in $\lambda K_{2 t+1}$ to be the unique value $d \in\{1,2, \ldots, t\}$, such that $u$ and $v$ differ modulo $2 t+1$ by $d$. Define values $v_{1}, v_{2}, \ldots, v_{2 t}$ (calculated modulo $2 t+1$ from the residues $0,1, \ldots, 2 t$ ) by

$$
v_{i}=-v_{t+i}= \begin{cases}i(-1)^{i+1}, & \text { for } 1 \leqslant i \leqslant\lceil t / 2\rceil \\ i(-1)^{i}, & \text { for }\lceil t / 2\rceil+1 \leqslant i \leqslant t .\end{cases}
$$

These values satisfy the following properties.

(P1) For each $v \in \mathbb{Z}_{2 t+1} \backslash\{0\}$ there is a unique $i \in\{1,2, \ldots, 2 t\}$ such that $v_{i}=v$.

(P2) For each $d \in\{1,2, \ldots, t\}$ there is a unique $i \in\{1,2, \ldots, t\}$ such that $v_{i}$ and $v_{i+1}$, as well as $v_{t+i}$ and $v_{t+i+1}$, differ modulo $2 t+1$ by $d$.

(P3) For each $d \in\{1,2, \ldots, t\}$ there is a unique $i \in\{1,2, \ldots, t\}$ such that $v_{i}$ and $v_{t+i}$ differ modulo $2 t+1$ by $d$.

In particular, property $(\mathrm{P} 1)$ allows us to think of these $v_{i}$ as alternative labellings for the non-zero vertices in $\lambda K_{2 t+1}$. Then $\mathcal{D}=\left\{\rho^{\alpha}\left(C_{i}\right) \mid 0 \leqslant \alpha \leqslant 2 t\right.$ and $\left.1 \leqslant i \leqslant t\right\}$, where

$$
C_{i}=\left(v_{i}, v_{i+1}, \ldots, v_{i+\mu-1}, 0, v_{t+i+\mu-1}, v_{t+i+\mu-2}, \ldots, v_{t+i}\right)
$$

for each $i \in\{1,2, \ldots, t\}$ and subscripts of $v$ are calculated modulo $2 t$ from the residues $1,2, \ldots, 2 t$, is a $\lambda$-cycle decomposition of $\lambda K_{2 t+1}$ (see [10] for more detail). We use this decomposition $\mathcal{D}$ to deal with cases (i) and (ii) separately. 
(i) For each $i \in\{1,2, \ldots, t\}$ we label the edges of the cycle $C_{i}$ so that the edge $v_{t+i} v_{i}$ has label 0 , the edge $v_{i} v_{i+1}$ has label 1 , the edge $v_{i+1} v_{i+2}$ has label 2 and so on, up to the edge $v_{t+i+1} v_{t+i}$ which has label $\lambda-1$. It is clear from properties (P1), (P2) and (P3) above, that for each $d \in\{1,2, \ldots, t\}$ and each $\ell \in\{0,1, \ldots, \lambda-1\}$, there is a unique $i \in\{1,2, \ldots, t\}$ for which the cycle $C_{i}$ contains an edge of difference $d$ with label $\ell$. Taking advantage of this fact, we extend this labelling to the edges of each cycle in $\mathcal{D}$ by assigning, for each $\alpha \in\{1,2, \ldots, 2 t\}$ and each $i \in\{1,2, \ldots, t\}$, the edge $\rho^{\alpha}(u) \rho^{\alpha}(v)$ in cycle $\rho^{\alpha}\left(C_{i}\right)$ the same label as the edge $u v$ in cycle $C_{i}=\rho^{0}\left(C_{i}\right)$. The resulting edge labelling thus induces a $\lambda$-weight function $\omega$ on $\mathcal{D}$. It remains to show that $\omega$ is balanced.

Let $\vec{K}_{2 t+1}$ be the directed version of $K_{2 t+1}$ with directed edge-set

$$
E=\{(v, v+d) \mid 0 \leqslant v \leqslant 2 t \text { and } 1 \leqslant d \leqslant t\},
$$

where $v+d$ is calculated modulo $2 t+1$ from the residues $0,1, \ldots, 2 t$. Hence $\vec{K}_{2 t+1}$ is invariant under the permutation $\rho$ and we need only show that, for each $i \in\{1,2, \ldots, t\}$, the sum-weight (with respect to $\vec{K}_{2 t+1}$ ) of the cycle $C_{i}$ is a multiple of $\lambda$. We do this as follows.

Orient the edges of the cycle $C_{i}$ to form the directed cycle

$$
\vec{C}_{i}=\left(v_{i}, v_{i+1}, \ldots, v_{i+\mu-1}, 0, v_{t+i+\mu-1}, v_{t+i+\mu-2}, \ldots, v_{t+i}\right)_{D} .
$$

Recall that for each $\ell \in\{0,1, \ldots, \lambda-1\}$ there is precisely one edge in $C_{i}$ labelled $\ell$. Since the edge labelled 0 contributes nothing to the sum-weight of $C_{i}$, we need only show that for each $\ell \in\{1,2, \ldots, \mu\}$ (recall that $\lambda=2 \mu+1$ ), if $e_{1}$ and $e_{2}$ are the directed versions (in $\vec{C}_{i}$ ) of the edges labelled $\ell$ and $\lambda-\ell$ in $C_{i}$, then either $e_{1}$ and $e_{2}$ both belong to $E$, or neither $e_{1}$ nor $e_{2}$ belongs to $E$. (Hence their contribution to the sum-weight of $C_{i}$ will be either $\lambda$ or $-\lambda$.) Consider first the directed versions (in $\vec{C}_{i}$ ) of the edges labelled $\mu$ and $\mu+1$ in $C_{i}$; that is, the directed edges $\left(v_{i+\mu-1}, 0\right)$ and $\left(0, v_{t+i+\mu-1}\right)$ respectively. By definition, $v_{t+i+\mu-1} \equiv-v_{i+\mu-1}(\bmod 2 t+1)$. Hence

$$
0-v_{i+\mu-1} \equiv v_{t+i+\mu-1}-0 \quad(\bmod 2 t+1)
$$

and the result follows by the definition of $E$. Similarly, for each $\ell \in\{1,2, \ldots, \mu-1\}$, the directed versions (in $\vec{C}_{i}$ ) of the edges labelled $\ell$ and $\lambda-\ell$ in $C_{i}$ are $\left(v_{i+\ell-1}, v_{i+\ell}\right)$ and $\left(v_{t+i+\ell}, v_{t+i+\ell-1}\right)$ respectively. By definition, $v_{t+i+\ell} \equiv-v_{i+\ell}(\bmod 2 t+1)$ and $v_{t+i+\ell-1} \equiv$ $-v_{i+\ell-1}(\bmod 2 t+1)$. Hence

$$
v_{i+\ell}-v_{i+\ell-1} \equiv v_{t+i+\ell-1}-v_{t+i+\ell} \quad(\bmod 2 t+1),
$$

and the result follows by the definition of $E$.

(ii) Note that in this case we assume that $\mu \geqslant 2$. For each $i \in\{1,2, \ldots, t\}$ label the edges of the cycle $C_{i}$ as in the proof of (i) above, and define $D_{i}$ to be an exact copy of the edge-labelled cycle $C_{i}$. Hence $\left\{\rho^{\alpha}\left(C_{i}\right), \rho^{\alpha}\left(D_{i}\right) \mid 0 \leqslant \alpha \leqslant 2 t\right.$ and $\left.1 \leqslant i \leqslant t\right\}$ is a $\lambda$-cycle decomposition of $2 \lambda K_{2 t+1}$. Moreover, for each $d \in\{1,2, \ldots, t\}$ and each 
$\ell \in\{0,1, \ldots, \lambda-1\}$, there is a unique $i \in\{1,2, \ldots, t\}$ for which the cycles $C_{i}$ and $D_{i}$ each contain a copy of the same edge of difference $d$ with label $\ell$.

Now, for each $i \in\{1,2, \ldots, t\}$ the cycles $C_{i-\mu+1}$ and $D_{i-\mu+1}$ (with subscripts calculated modulo $t$ from the residues $1,2, \ldots, t)$ each contain the 2-path $\left[v_{i}, 0, v_{t+i}\right]$. Moreover, the edges of this 2-path, namely $v_{i} 0$ and $0 v_{t+i}$, each have difference $i$, and are labelled $\mu$ and $\mu+1$ respectively in both $C_{i-\mu+1}$ and $D_{i-\mu+1}$. For each $i \in\{1,2, \ldots, \mu\}$ we "modify" the cycle $C_{i-\mu+1}$ by removing this 2-path, hence forming the $(\lambda-2)$-path

$$
L_{i-\mu+1}=\left[v_{t+i}, v_{t+i-1}, \ldots, v_{t+i-\mu+1}, v_{i-\mu+1}, v_{i-\mu+2}, \ldots, v_{i}\right] .
$$

Thus in total we remove one edge of each difference $d \in\{1,2, \ldots, \mu\}$ labelled $\mu$, and one edge of each difference $d \in\{1,2, \ldots, \mu\}$ labelled $\mu+1$, from the cycles $C_{i-\mu+1}, 1 \leqslant i \leqslant \mu$. We do the same, this time for each $i \in\{2,3, \ldots, \mu\}$, to the cycle $D_{i-\mu+1}$, leaving the $(\lambda-2)$-path

$$
P_{i-\mu+1}=\left[v_{t+i}, v_{t+i-1}, \ldots, v_{t+i-\mu+1}, v_{i-\mu+1}, v_{i-\mu+2}, \ldots, v_{i}\right]
$$

in each case. Thus in total we remove one edge of each difference $d \in\{2, \ldots, \mu\}$ labelled $\mu$, and one edge of each difference $d \in\{2, \ldots, \mu\}$ labelled $\mu+1$, from the cycles $D_{i-\mu+1}$, $2 \leqslant i \leqslant \mu$. (Note that the subscripts for both $L_{i-\mu+1}$ and $P_{i-\mu+1}$ are also calculated modulo $t$ from the residues $1,2, \ldots, t$.) We then define two new $(\lambda-2)$-paths $G_{0}$ and $H_{0}$ on $2 \lambda K_{2 t+1}$ as follows (the reason for using this notation will soon become clear).

For each $j \in\{1,2, \ldots, \mu\}$ define

$$
x_{j}=\sum_{i=1}^{j}(-1)^{i+1}(\mu-i+1),
$$

and for each $j \in\{1,2, \ldots, \mu-1\}$ define $y_{j}=(2 t+1)-x_{j}$. Hence $x_{1}=\mu$ and, for each $j \in\{1,2, \ldots, \mu-1\}$, the values $x_{j}$ and $x_{j+1}$ differ by $\mu-j$. Similarly $y_{1}=(2 t+1)-\mu$ and, if $\mu>2$, then for each $j \in\{1,2, \ldots, \mu-2\}$ the values $y_{j}$ and $y_{j+1}$ differ by $\mu-j$. Then define

$$
G_{0}=H_{0}=\left[x_{\mu}, x_{\mu-1}, \ldots, x_{1}, 0, y_{1}, y_{2}, \ldots, y_{\mu-1}\right] .
$$

It is easy to see this is indeed a $(\lambda-2)$-path in $2 \lambda K_{2 t+1}$. We assign the edge $x_{\mu} x_{\mu-1}$ the label $\mu$ in $G_{0}$ and $\mu+1$ in $H_{0}$. We then assign each of the edges

$$
x_{\mu-1} x_{\mu-2}, x_{\mu-2} x_{\mu-3}, \ldots, x_{1} 0
$$

the label $\mu$ in both $G_{0}$ and in $H_{0}$; and we assign the remaining edges

$$
0 y_{1}, y_{1} y_{2}, \ldots, y_{\mu-2} y_{\mu-1}
$$

the label $\mu+1$ in both $G_{0}$ and in $H_{0}$. Hence $G_{0}$ contains one edge of each difference $d \in\{1,2, \ldots, \mu\}$ labelled $\mu$, and one edge of each difference $d \in\{2,3, \ldots, \mu\}$ labelled $\mu+1$. Similarly, the path $H_{0}$ contains one edge of each difference $d \in\{2, \ldots, \mu\}$ labelled $\mu$, and one edge of each difference $d \in\{1,2, \ldots, \mu\}$ labelled $\mu+1$. Thus together these 
two paths use edges of the exact same differences and labels as those we removed from the cycles $C_{i-\mu+1}, 1 \leqslant i \leqslant \mu$, and the cycles $D_{i-\mu+1}, 2 \leqslant i \leqslant \mu$. Moreover, since $\mu>1$, the paths $G_{0}$ and $H_{0}$ share at least two adjacent edges which also have the same labels in both $G_{0}$ and $H_{0}$.

Then for each $i \in\{1,2, \ldots, t\}$ we let $G_{i}$ be either the edge-labelled cycle $C_{i}$, if $C_{i}$ was not one of the "modified" cycles from above, or the edge-labelled path $L_{i}$ otherwise. Hence $t-\mu$ of the graphs $G_{i}$ are $\lambda$-cycles, and the remaining $\mu$ (plus the graph $G_{0}$ ) are $(\lambda-2)$-paths. Similarly, we let $H_{i}$ be either the edge-labelled cycle $D_{i}$, or the edge-labelled path $P_{i}$, depending on whether $D_{i}$ was one of the "modified" cycles. Hence $t-\mu+1$ of the graphs $H_{i}$ are $\lambda$-cycles, and the remaining $\mu-1$ (plus the graph $\left.H_{0}\right)$ are $(\lambda-2)$-paths. We note also that for each $i \in\{0,1, \ldots, t\}$ the graphs $G_{i}$ and $H_{i}$ share at least two adjacent edges which also have the same labels in both $G_{i}$ and $H_{i}$ (even in the one case where $G_{i}$ is a path and $H_{i}$ is a cycle).

Hence $\left\{\rho^{\alpha}\left(G_{i}\right), \rho^{\alpha}\left(H_{i}\right) \mid 0 \leqslant \alpha \leqslant 2 t\right.$ and $\left.0 \leqslant i \leqslant t\right\}$ is a decomposition of $2 \lambda K_{2 t+1}$ into

- $(2 t+1)(2 t-2 \mu+1)=n(n-\lambda+1)$ cycles of length $\lambda$; and

- $(2 t+1)(2 \mu+1)=n \lambda$ paths of length $\lambda-2$.

Moreover, suppose $\omega^{\prime}$ is the $\lambda$-weight function on this decomposition obtained by extending the edge labelling of each of the graphs $G_{i}$ and $H_{i}$ under the permutation $\rho$ (as in part (i) above). Then, under $\omega^{\prime}$, each cycle in the decomposition has sum-weight a multiple of $\lambda$ with respect to $\vec{K}_{n}$ (also defined above). Hence using the function $\omega^{\prime}$ we generate a decomposition, $\mathcal{D}^{\prime}$ say, of the graph $2\left(K_{n} * \bar{K}_{\lambda}\right)$ into $\lambda n(n-\lambda+1)$ gregarious $\lambda$-cycles and $n \lambda^{2}$ gregarious $(\lambda-2)$-paths (by associating vertices and labelled edges of $2 \lambda K_{n}$ with partite sets and matchings of $2\left(K_{n} * \bar{K}_{\lambda}\right)$ as in the proof of Lemma 2.6).

For each $i \in\{0,1, \ldots, t\}$ we let $X\left(G_{i}\right)$ (respectively, $X\left(H_{i}\right)$ ) be the set of $\lambda n$ cycles, or paths, in $\mathcal{D}^{\prime}$ generated by the graphs $\rho^{\alpha}\left(G_{i}\right)$ (respectively, $\rho^{\alpha}\left(H_{i}\right)$ ), $0 \leqslant \alpha \leqslant 2 t$. Then, by the remarks above, we can pair up the graphs in $X\left(G_{i}\right)$ with those in $X\left(H_{i}\right)$ so that the graphs in each pair share two adjacent edges. Finally we note that if $G_{i}$ (respectively, $H_{i}$ ) is a path, then due to the cyclic nature of the decomposition of $2 \lambda K_{n}$, each vertex in $2\left(K_{n} * \bar{K}_{\lambda}\right)$ appears as an endpoint of exactly two paths in the set $X\left(G_{i}\right)$ (respectively, $X\left(H_{i}\right)$ ). Since there are precisely $\mu+1$ graphs $G_{i}$ which are paths, say $G_{\alpha_{1}}, G_{\alpha_{2}}, \ldots, G_{\alpha_{\mu+1}}$, and $\mu$ graphs $H_{i}$ which are paths, say $H_{\alpha_{\mu+2}}, H_{\alpha_{\mu+3}}, \ldots, H_{\alpha_{\lambda}}$, we can thus add an extra partite set of size $\lambda$ to our graph $2\left(K_{n} * \bar{K}_{\lambda}\right)$, say $\left\{\infty_{1}, \infty_{2}, \ldots, \infty_{\lambda}\right\}$, and attach the ends of each path in the sets $X\left(G_{\alpha_{1}}\right), \ldots, X\left(G_{\alpha_{\mu}}\right), X\left(H_{\alpha_{\mu+1}}\right), \ldots, X\left(H_{\alpha_{\lambda}}\right)$, to the vertices $\infty_{1}, \infty_{2}, \ldots, \infty_{\lambda}$ respectively. Thus these form gregarious $\lambda$-cycles in each case. Note that every vertex in the part $\left\{\infty_{1}, \infty_{2}, \ldots, \infty_{\lambda}\right\}$ is attached to every vertex in the graph $2\left(K_{n} * \bar{K}_{\lambda}\right)$ twice. Hence we have a decomposition of the graph $2\left(K_{n+1} * \bar{K}_{\lambda}\right)$ into gregarious $\lambda$-cycles. Moreover, since we haven't affected the underlying graphs from our decomposition of $2\left(K_{n} * \bar{K}_{\lambda}\right)$, we can partition the cycles in this decomposition into pairs in such a way that the cycles in each pair share two adjacent edges. This completes the proof.

We are now ready to present the main result of this section. 
Proof of Theorem 1.1 (ii) Let $n, m$ and $k$ satisfy the conditions of the theorem. We then split the problem according to whether $n$ is odd or even.

Case I. Suppose $n$ is odd. Since $n$ and $k$ are both odd with $n \geqslant k \geqslant 3$, there exists a gregarious $k$-cycle decomposition of $K_{n} * \bar{K}_{k}$ by Lemma 4.1 (i) and Lemma 2.6. The result then follows by Lemma 3.5.

Case II. Suppose $n$ is even. If $k=3$ the result follows by Hanani [5]. Suppose then $k \geqslant 5$. Since $n$ is even and $n>k \geqslant 5$, there exists a gregarious $k$-cycle decomposition of $K_{n} * \bar{K}_{2 k}$ by Lemma 4.1 (ii) and Lemma 2.2. Moreover, since $n$ is even, $m$ is also even and hence $m \equiv 0(\bmod 2 k)$. The result then follows by Lemma 3.5.

\section{References}

[1] B. R. Alspach and H. J. Gavlas, Cycle decompositions of $K_{n}$ and $K_{n}-I, J$. Combin. Theory Ser. B 81 (2001), 77-99.

[2] E. J. Billington and D. G. Hoffman, Equipartite and almost-equipartite gregarious 4-cycle systems, Discrete Math. 308 (2008), 696-714.

[3] E. J. Billington, D. G. Hoffman and C. A. Rodger, Resolvable gregarious cycle systems of complete equipartite graphs, Discrete Math. 308 (2008), 2844-2853.

[4] E. J. Billington, D. G. Hoffman and B. R. Smith, Equipartite gregarious 6- and 8-cycle systems, Discrete Math. 307 (2007), 1659-1667.

[5] H. Hanani Balanced incomplete block designs and related designs, Discrete Math. 11 (1975), 255-369.

[6] J. Liu, A generalization of the Oberwolfach problem and $C_{t}$-factorizations of complete equipartite graphs, J. Combin. Designs 8 (2000), 42-49.

[7] J. Liu, The equipartite Oberwolfach problem with uniform tables, J. Combin. Theory Series A, 101 (2003), 20-34.

[8] M. Šajna, Cycle decompositions III: Complete graphs and fixed length cycles, $J$. Combin. Designs 10 (2002), 27-78.

[9] B. R. Smith, Equipartite gregarious 5-cycle systems and other results, Graphs and Combinatorics 23 (2007), 691-711.

[10] B. R. Smith, Cycle decompositions of complete multigraphs, J. Combin. Designs, (to appear).

[11] B. R. Smith, Decomposing complete equipartite graphs into odd square-length cycles: number of parts odd, (submitted). 